\title{
USING CONCEPT MAPS FOR ONTOLOGY DEVELOPMENT: A CASE IN THE WORK ORGANIZATION DOMAIN
}

\author{
António Soares* and Cristóvão Sousa** \\ * Faculty of Engineering of the University of Porto, \\ Electrical and Computer Engineering Department (FEUP/DEEC) and INESC Porto \\ e-mail: als@fe.up.pt \\ ** Institute for Systems and Computer Engineering of Porto (INESC PORTO) \\ e-mail: cpsousa@inescporto.pt
}

\begin{abstract}
Ontologies are a technological key factor regarding the knowledge management domain. This paper presents a graphical-based knowledge representation approach using concept maps towards creating a formal work organization ontology, which was translated into a Content Management System in order to manage the work design information. Some aspects related with the advantages of visual approaches for collaborative development of ontologies are discussed.
\end{abstract}

\section{INTRODUCTION}

Organizational performance depends on process efficacy and quality of life at work. A common understanding of how work should be realized based on an effective management of high skills, high trust and high quality is a key factor for business process efficacy, which demands for new forms of work organization. The implementation of new forms of work organization is a complex development process that implies an interaction between work design, individuals and groups.

Technical and organizational innovation processes e.g., business process reengineering and work redesign are normally loosely coupled leading to a sub-optimal socio-technical system. Closer interaction between these design processes is needed which calls for multidisciplinary development teams sharing knowledge and competencies. Multidisciplinary cooperation is a new challenge and a new category of methodological tools supported by modern knowledge management technologies is needed in order to make feasible such complex development processes. In this context ontologies play a key role by providing a shared conceptual model of a specific domain, that means, it provides the vocabulary which can be used in the work organization domain, which entails the type of objects and concepts that exist and their properties and relations.

The goal of this paper is the characterization of work within and between organizations regarding the organizational concepts, types of relationships and the new trends of work organization through the use of ontologies' engineering. An approach to collaborative work design of individuals and groups, using graphical-based tools will be adopted. 
This paper is organized as follows. In the next section some visual approaches for graphical representation of ontologies will be briefly presented. Section three focuses an approach to the knowledge representation through the creation of ontologies with conceptual maps. The fourth section presents the work organization ontology developed with concept maps in the CmapTools COE authoring environment. Section five discloses a practical and possible application scenario of the developed ontology. All the steps needed to build a hierarchically set of folders and files into a Content Management System (CMS) according to the ontology exported in OWL, are described. Some directions for further work are addressed in section six.

\section{VISUAL APPROACHES FOR ONTOLOGY DEVELOPMENT}

Ontological level of knowledge representation is based in conceptualization. Conceptualization comprises a simplified vision of the objects, concepts, and other entities that are assumed to exist in some area of interest and the relations among them (Gruber, 1993). The use of graphical knowledge representation formalisms with a representational vocabulary agreement of terms of conceptualization of the universe of discourse is a new high potential approach in the knowledge management context. Semantic networks, conceptual graphs, rdf, topic maps and concept maps are the graphical knowledge representation formalisms briefly presented, which may also be used for ontology development.

Visually, a semantic network is a set of nodes which represent concepts and instances, connected by arcs which represent relations between nodes. According to (Gordon, 2000), semantic networks are a powerful knowledge representation system because they are easy to understand by humans and can be used in automated processing systems which means that they can also became a vehicle to archive organization knowledge. While visual tools for ontology construction, semantic networks provide the conceptual representation of a domain allowing the explicit representation of concepts, relations and instances. Semantic networks have also mechanisms for formal representation of knowledge. However, the interpretation of a semantic network may cause some confusion. No distinction is made between different types of links and the distinction between concepts and objects is not clear (Baader, 1999). Due its lack of formal semantic characteristic, there are many variances of semantic networks.

Conceptual Graphs (CGs), by its turn, is a formal logic-based knowledge representation developed by John Sowa. CGs are, in fact, a variation of semantic network combined with logic! CGs are a very powerful and versatile tool for knowledge representation. They are human readable and machine process able. Nevertheless it is not possible to draw a CG without having a basic knowledge of logic and CGs itself.

Resource Description Framework (RDF) is a framework for representing information in the web. RDF has the capability to formal express the data meaning allowing interoperability and provides an integration environment between different patterns of metadata $^{1}$. RDF language was created to represent a simple data model based on XML and using vocabulary based on Uniform Resource Locator (URL). The data model is graphically represented through triples which consist in three types of objects that describe relationships between resources regarding properties and values. In terms of ontology representation, RDF allows the explicit representation of resources (concepts), properties and statements. RDF expresses the meaning of data allowing interoperability in the web. It is therefore a knowledge representation formalism which provides the structure

\footnotetext{
${ }^{1}$ htpp://www.w3c.org/TR/rdf-concepts
} 
that is used to represent data models for objects and their relations. However it needs RDF Schema in order to provide mechanisms to declare properties and define relations between properties and resources. RDF Schema is used to describe, semantically, properties, classes of web resources and the type of data for the property values. It extends RDF with new vocabulary allowing the knowledge to be represented through ontologies.

Regarding Topic Maps (TM), they have a great expressive power. In some sense they are a reformulation of semantic networks and conceptual graphs. Additionally they offer a new and standard way of encoding and exchanging knowledge. Technically a TM is formed by three concepts: topic name, association and occurrence. A Topic can be everything - an object, as person, a concept, etc. The association indicates how a topic is related with other topics. Each topic involved in an association, is said to play a rule (Pepper, 2000). For example: from an Organizational point of view, "Work organization defines work practices". In this case there are two topics - WorkOrganization and WorkPractice and there is an association between them, where WorkOrganization plays the rule of the one that defines the WorkSystem. In terms of ontology representation TM can represent facts, procedures, concepts and complex relations between concepts and real world occurrences. It is possible to represent knowledge in a formal way. However, and despite of TM's flexibility, they are very difficult to manage.

Conceptual Maps (Cmaps), are able to represent meaningful relationships between concepts linked by words to from a semantic unit (Canãs, 1999). The concepts are included in circles or boxes while relations between concepts are represented by links connecting the boxes. The links are labelled, describing the relation between two concepts. Propositions result from the phrases composed by the concepts and the link label (concept - verbal phrase - concept). According to (Garcia et al., 2006) Cmaps are very useful in facilitating the visualization and discussion, and in providing domain experts with a tool that could be used to declare the primary elements of their knowledge. Cmaps' simplicity and explicitly make them very useful in several areas namely: Knowledge organization and creation; Collaborative learning; Domain summarization; Browsing tool.

\section{USING CONCEPT MAPS FOR ONTOLOGY DEVELOPMENT}

Regarding the use of Cmaps for ontology development it was analyzed the ontology development process (shown in figure 1) and ontology development requirements in order to evaluate the suitability of Cmaps as ontology development tool.

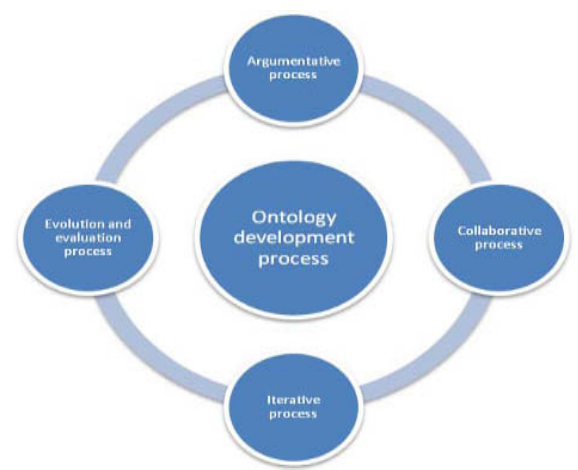

Figure 1. Ontology development process 
Ontology development is not a static process but an iterative task that crosses several stages such as: Argumentative process; Collaborative process; Iterative process; Evolution and evaluation process. This means that when creating an ontology, domain experts and knowledge engineers argue about terms and relationships (Stevens, 2000). Therefore collaborative interaction of arguments increase the amount of information attached to the concepts or relationships (Stevens, 2000). The arguments derived from discussions can be commentaries, files or even other maps. In general, discussions take place at forums, email messages, chats, etc, and become necessary for knowledge engineer to filter the messages and formalize that knowledge. This task is certainly hard to perform and, for sure, not all information can be retrieved.

By other hand methodologies or tools for ontology building must address Collaboration Support and Argumentative Support according to the ontology development process. According to (Gruber, 2006), domain experts are the central performer on ontology elicitation process, because they have the experience and knowledge about a specific area. Grounding support can be achieved in two ways. Methodologies and tools can use fundamental design patterns of foundational ontologies to provide guidance to domain experts. The second approach is to use a foundational ontology as a base model for the definition of more specific domain (Gruber, 2006). Cognitive support is used to leverage innate human abilities, such as visual information processing, to increase human understanding and cognition of challenging problems (Walenstein, 2002). As argued in (Gruber, 2006), cognitive support for ontology development is required in order to facilitate the useful/exchange of information and at the same time record the entire process. In terms of ontology development, Cmaps allow explicit specification of concepts, relations, constraints, prepositions. Allow explicit representation of consensual knowledge and are very good for conceptualization enhancing cognitive support. Even though Cmaps only allow the representation of informal knowledge, CmapTools Ontology Editor (COE) software provides via Cmaps a complete collaborative and argumentative environment, based on graphical direct manipulation and representation to facilitate the continuous exchange of information among domain experts. COE was developed in order to give CmapTools more interoperability and formalized notations meeting ontological agreements. $\mathrm{COE}$ allows the capture of knowledge structures using templates which can be dropped directly onto ontology map. The templates are graphical representations for commonly used OWL structures. With COE, the lack of Cmaps capability for representing formalized knowledge is solved in spite of some interoperability problems still remaining. Combining Cmaps with $\mathrm{COE}$, which provide collaborative and argumentative support and formal knowledge representation based on templates, we have an interesting approach for ontology development. This particularly approach was selected to be followed in the work organization ontology development presented in the next section

\section{A WORK ORGANIZATION ONTOLOGY}

The work organization ontology was developed in the context of a national project called COllaboration and DEmocratic work design in Virtual Organizations (CODEwork@vo). CODEwork project joined sociologists and engineers in an attempt to find out a work organization model considering both technical and social perspective. A visual approach was needed due the heterogeneity of backgrounds of the experts in domain conceptualization and ontology development. At the same time, mechanisms of communication, discussion and argumentation were desirable which leaded to the 
selection of CmapTools COE to support the creation of the ontology in the work organization domain.

The ontology development in the work organization domain had several steps; the next figure shows the process that we perform for creating the ontology.

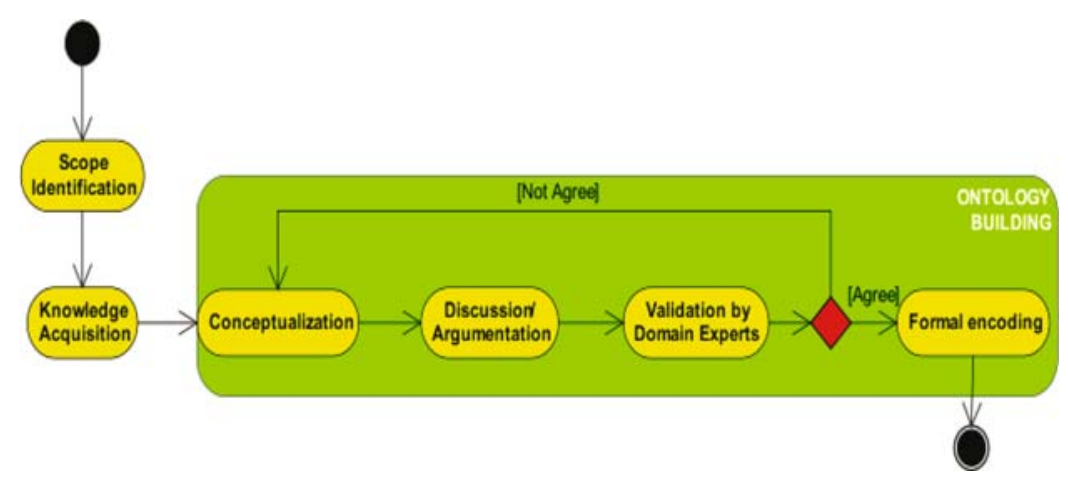

Figure 2. Ontology development methodology

The first phase was the definition of the ontology scope. Knowledge acquisition was the next phase, where all terms about the domain within the scope range were collected. Explicit definitions and descriptions were made and the competency questions were formalized in order to see if our ontology fulfilled the main scope. Later, Cmaps were created freely, without any formal restriction. The created Cmaps represented only a particular view of the problem. The next steps were to put the map on the server for collective discussion and validation. The team argued about the conceptual map trough notes, forum or other maps, files, links..., until it is reached a common understanding. After the cmap had been validated, it was converted in a formal notation. This formal notation was achieved by using COE templates.

\subsection{Ontology goal and scope}

The main goal of the work organization ontology is to provide a better understand of work organization design process, in order to better refine organization orientation strategies, helping employees with some decision making roles. At the same time provide a common understanding about work, avoiding communication gaps among individuals in an environment where the organizations are increasingly dependent from each other. The ontology scope is focused in the understanding of the main concepts related with the process of work design and organizational design in a human resource management perspective within and between organizations.

\subsection{Ontology high level description}

Work organization can be defined as a multilevel concept, divided in 3 levels of context: External Context, Organizational Context, and Work Context (Sauter et al., 2002). We cannot do much about external context. The most immediate thing we can do is to predict what will happen next and act according to it. The main management challenge is to organize/optimize all work and resources of an organization in order to endow enterprises the ability to easily adapt to market changes. The figure 3, presented below, shows the conceptualizations of work organization in a concept map. That cmap emphasize the following assumptions: 
1. Work Organization can be defined as the result of work design process or organizational design process implementations.

2. Work organization has a direct impact over people and their skills, the processes and the overall work system ${ }^{2}$, in view of the fact that it refers to the (re)organization of those concepts.

3. Regarding work design, we can consider two main different scenarios according to the boundaries of work system, thus we can have a work design process based in a vertical division of work, which the components of work are distributed inside organization among one or more hierarchical levels (Sinha, 2005). By the other hand we have the case in which the components of work are distributed across many organizational units of one or many firms and, in these case, we have a work design process based in a scenario of vertical division of work ( Knublauch et al., 2004).

4. Organizational design has a set of structural dimensional, structural determinants and design models that drive the implementation process in an organization.

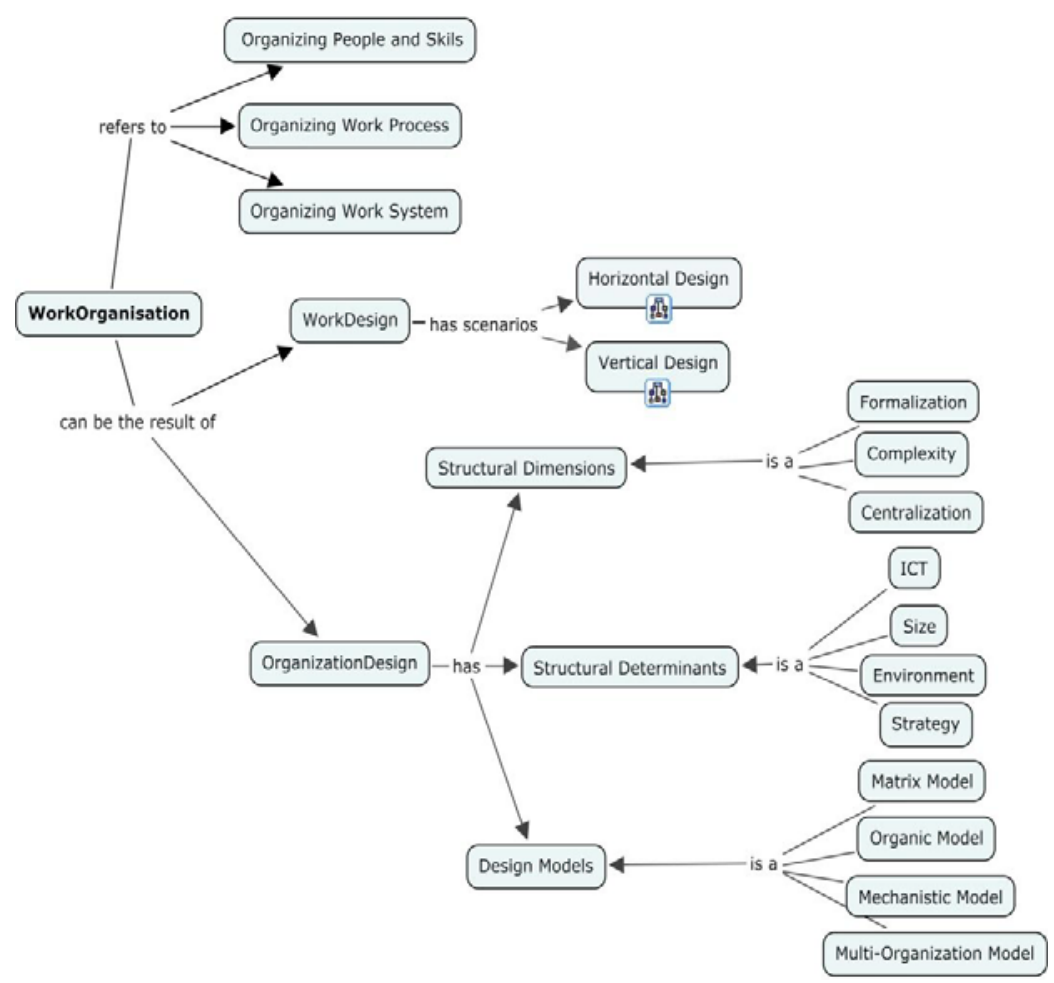

Figure 3. Work Organisation conceptualisation

In order to encode the ontology, COE software was used and the result is shown in figure 4 .

${ }^{2}$ Work system is composed be 5 elements: individuals, tasks, tools and technologies. 


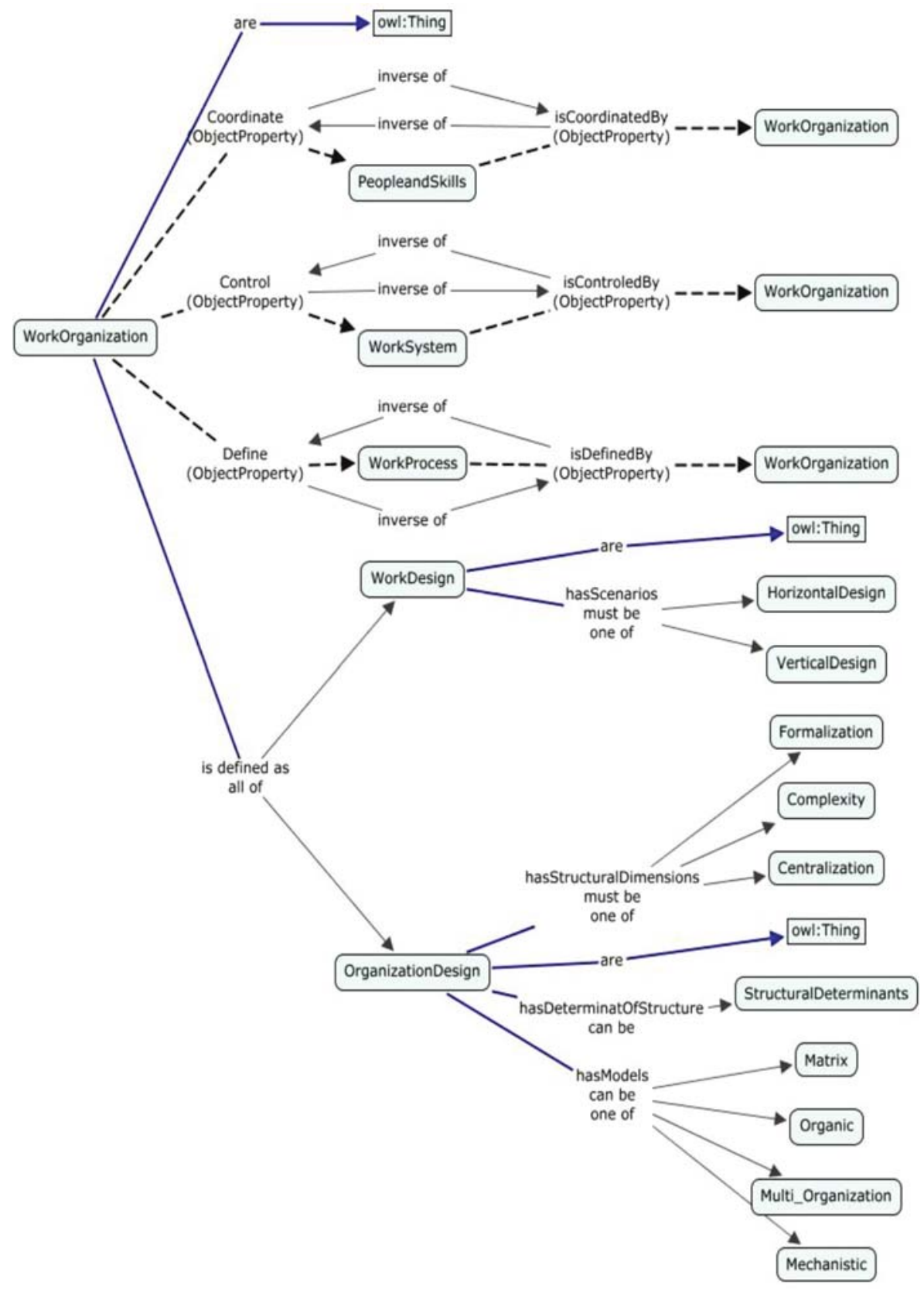

Figure 4. Work Organisation ontology map (partial map) 


\section{APPLICATION SCENARIO: MANAGING WORK DESIGN INFORMATION}

Technically, information management can be performed through the implementation of content management systems (CMS). CMS are practical translations of Knowledge Management and Information Management approaches.

In this section we will describe how to set up Plone CMS content based on OWL ontologies. The objective is to reach a simple manageable hierarchical distribution of folders and files trough a web portal, according to the concepts and relations, which compose the work organization ontology. The steps performed to achieve that goal are presented in the figure 5 .

Step 1: Development of work organization ontology in COE.

Step 2: Convert owl ontology to UML trough the Protégé UML plug-in and create UML composition relations. In OWL it is not possible to specify composition relations. With Protégé UML plug-in we specified that JobAnalysis concept was composed by three other concepts namely: JobSpecification, JobDescription and JobPerformane, then we transformed the ontology into and UML project.

Step 3: This step is optional. We opened the project with ArgoUML in order to see if the UML model converted within Protégé is consistent. At this step we may apply stereotypes specifying which object types (e.g. folder, file, special folder, discussion thread, image, etc.) our classes will assume within Plone.

Step 4/5: Using ArchGenXML utility, our UML model is converted into a valid Plone product which can be installed trough Plone administrator interface.

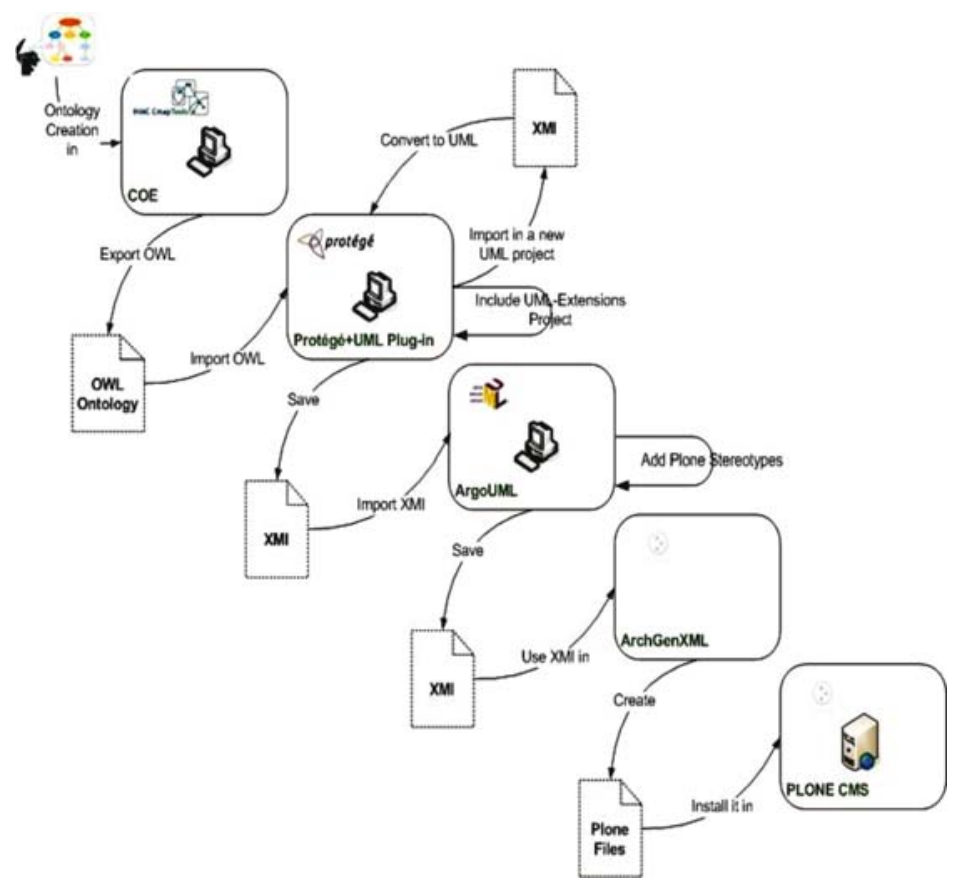

Figure 5. Application Scenario Steps 
As result of the previous steps, we obtained a web portal where we are able to manage work design information according with the concepts and relations figured in the ontology.

\section{CONCLUSION AND FURTHER WORK}

The knowledge is not only in the ontology engineers heads, thus it is highly recommended the specification of a knowledge model supported by simple knowledge representation formalisms that allow domain specialists to discuss together about a certain domain in order to be obtained a set of concepts and its relations according with the experience, know-how and surveys of the right people without having the concern about knowledge representation technical issues. In the first step of knowledge acquisition, Cmaps do fairly the job due its informal characteristics. However, an easy knowledge formalism is not sufficient by itself to support the construction of a knowledge model. Thence came into existence the CmapTools Software by IHMC providing a complete collaborative knowledge representation environment based on CMs.

By definition ontology has formal constraints, so it is necessary to transform cmaps into a formal notation. A framework that implements a solid methodology for cmaps translation into COE formal conventions with validation mechanisms is needed.

We also foresee cmaps and Ontologies as a solution for today's' new programming challenges. Nowadays, all people access to the internet to rapidly retrieve information from several different sources either for professional or personal use. This new situation asks for new ways to develop and present web content. A possible future approach could be based on concept maps as a tool to set up a CMS for implementing e.g., a web portal. At the same time, it would be interesting to have the reverse synchronous standard mechanism in which the content created in a CMS could be used to maintain and evaluate ontologies(e.g., delete/add new instances to the ontology). Interoperability Semantic Web could be the answer at a short/medium term. 


\section{REFERENCES}

1. Gruber TR. Toward principles for the design of ontologies used for knowledge sharing. In Nicola Guarino, Ed., International Workshop on Formal Ontology, Padova, Italy, 1993.

2. Gordon JL."Creating Knowledge Maps by Exploiting Depend Relationships", Knowledge Based Systems 2000; vol. 13: 71-79.

3. Baader F. "Logic-Based Knowledge Representation," in Artificial Intelligence Today: Recent Trends and Developments, vol. 1600/1999, Lecture Notes in Computer Science: Springer Berlin / Heidelberg, 1999 pp. 13.

4. Pepper S. "The TAO of Topic Maps," presented at XML Europe 2000, Paris, France, 2000.

5. Garcia A, Norena A, Betancourt,Ragan M. "Cognitive support for an argumentative structure during the ontology development process," in 9th Intl. Protégé Conference. Standford, California, 2006.

6. Stevens R, Goble C, Bechhofer S. "Ontology-based Knowledge Reresentation for Bioinformatics," Briefings in Bioinformatics 2000; vol. 1: 398-416.

7. Gruber A, Westenthaler R, Gahleitner E. "Supporting domain experts in creating formal knowledge models (ontologies)," presented at I-Know'06: 6th International Conference on Knowledge Management, Graz, Austria, 2006

8. Walenstein A, "Foundations of cognitive support: toward abstract patterns of usefulness." In: 14th Annual Conference on Design, Specification, and Verification of Interactive Systems (DSV-IS'2002), Rostock, Germany, 2002.

9. Sauter S, Brightwell W, Colligan M, Hurrell J, LeGrande D, Lessin N, Lippin R, Lipscomb J, Murphy, L, Peters R, Keita G, Robertson S, Stellman J, Swanson N, Tetrick L. The Changing Organization of Work and the Safety and Health of Working People, Knowledge Gaps and Research Directions, National Institute for Occupational Safety and Health, 2002, pp. 1-43.

10. Sinha K, Van de Ven A, Designing Work Within and Between Organizations, Frontiers of Organization Science, Laguna Beach, CA, 2005.

11. Knublauch H, Fergerson R, Noy N, Musen M. "The Protégé OWL Plugin: An Open Development Environment for Semantic Web Applications", 3rd International Semantic Web Conference, Hiroshima, Japan, 2004. 